\title{
Managing Variable Transmittance Windowpanes by Model-Based Autonomous Control
}

\author{
Sotirios D. Kotsopoulos ${ }^{1}$, Federico Casalegno ${ }^{1}$, Massahiro Ono $^{2}$ and Wesley Graybill ${ }^{2}$ \\ 1. Department of Comparative Media Studies, School of Humanities Arts and Social Sciences, Massachusetts Institute of Technology, \\ Cambridge MA 02139, USA \\ 2. Department of Computer Science, Massachusetts Institute of Technology, Cambridge MA 02139, USA
}

\begin{abstract}
The use of digitally activated, variable transmittance materials and artificial intelligence methods to building control will enhance the performance of buildings, and programmable components will change the traditional modes of architectural design, manufacturing and construction. In the presented key study, the architectural form and functionality of windows are revisited with a view to integrate current advances in material science, control systems engineering and human-computer interaction. The features of a building facade, involving a matrix of programmable windows that enables precise control of daylight, view and privacy in the interior of a house are discussed. Managing the variable transmittance materials of the facade by an autonomous high-level control system allows the optimization of the house performance based on real time data and the schedule of the inhabitants. Using constraint violations as a measure of success, the autonomous control of the house outperforms any existing deterministic control models.
\end{abstract}

Key words: Variable transmittance materials, autonomous control, programmable solar facade.

\section{Introduction}

Advancements in material science, control systems engineering and human-computer interaction can give rise to new conceptions of architecture, where people will engage in novel experiences. This paper discusses how the integration of variable transmittance materials and AI (artificial intelligence) methods to building control can revolutionize the architecture of buildings. The benefits from this integration can be multifaceted. Using the form and the fabric of the building envelope as a filter of the exterior environment, while providing the capacity to modify the attributes of specific architectural components in real time, can have far reaching implications both in energy efficiency and in building aesthetics.

This study presents the material and control features of a specific dynamic architectural component, a "programmable window" that was designed for a

Corresponding author: Sotirios D. Kotsopoulos, Ph.D., research associate, research fields: sustainable architecture, programmable materials engineering, building performance simulation and control. E-mail: skots@mit.edu. prototype house, in Trento, Italy (Fig. 1). The form and the functionality of traditional windows are revisited with a view to integrate variable transmittance materials and autonomous control. The south facade of the prototype house in Trento is a reconfigurable solar wall made of programmable windows that incorporate variable transmittance materials. The programmable windows can be tinted to a spectrum of opacities or opened to precise angles, enabling precise adjustment of temperature, airflow, solar radiation, view and privacy at the house interior. Proper control of the windows can lead to significant reduction in heating and cooling needs thus reducing the operation of the HVAC (heating ventilating and air conditioning) system. A risk-sensitive autonomous control system manages the performance of the windows and of the building envelope, and provides maximum comfort while minimizing energy use. The control system optimizes the long-term energy consumption of the house, based on real-time sensory data and the schedules of the inhabitants. 


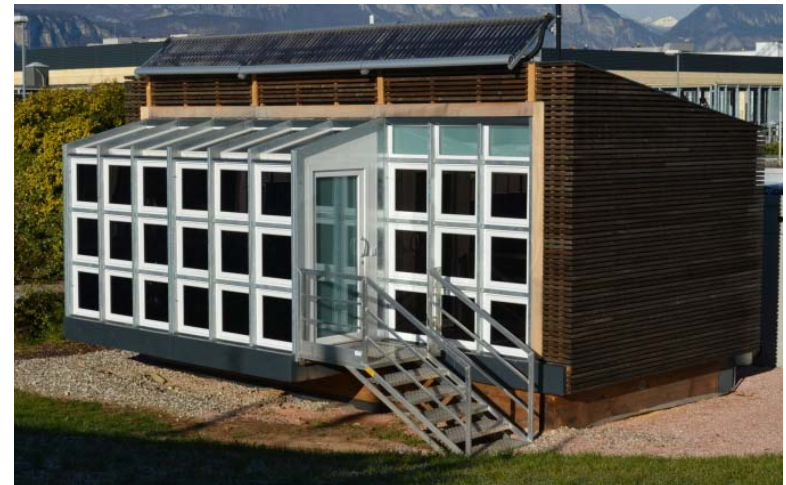

Fig. 1 The "dynamic facade" of the prototype house in Trento, Italy, oriented towards south.

In general, a window is a transparent or translucent opening in a wall or a door, allowing selective admission of light air and sound. Windows are usually glazed or covered in some transparent or translucent material like float glass. They are held in place by frames, which prevent them from collapsing and they can be un-operable or operable. Windows are important building components because they have social, aesthetic and cultural associations. They play an essential role not only in the adjustment of the interior lighting and thermal conditions, but also in the relationship between interior and exterior. Ordinary glass windows became common in homes only in the early 17th century, while modern-style floor-to-ceiling windows became possible after the industrial glass-making process was perfected.

Today, variable transmittance materials and AI methods to building control can add new dynamics to the evolution of built environments, including the adaptation of their aesthetic presence and performance based on real time conditions. Programmable windows can take active role in the dynamic optimization of building performance with low operational cost, and contribute to the enhancement of living experience. By adjusting the state of electrochromic glass panes, the solar transmittance of windows becomes a programmable feature. This allows to regulate with precision the admittance of solar radiation and heat and to control the levels of interior illuminance and thermal comfort [1, 2]. Hence, the efficient management of the transitions of electrochromic windows can be a factor in reducing energy consumption [3]. Furthermore, the use of PDLC (polymer dispersed liquid crystal) films and suspended particle displays can eliminate the need for traditional blinds and shutter systems to renew building aesthetics.

In combination with the idea of autonomous control of the envelope and systems, this approach can produce buildings that are energy saving, comfortable and architecturally original. Model-based control systems promise to adapt the functioning of house devices to precisely match user comfort needs. While the traditional PID (proportional integral derivative) control systems minimize operational error deterministically, based on a given set point, model-based control systems can manage the interior atmosphere by acknowledging particular preferences, and flexible scheduling by the inhabitants. Hence, while standard HVAC systems receive a single input temperature and operate in reactive mode to maintain this temperature overtime, model-based systems are capable of addressing the full spectrum of user needs. Buildings equipped with such capacities can provide new ways to think about the management of energy, daylight and privacy, and drastically transform the way the authors design, perceive and inhabit the built environment [4].

An example in deploying these technologies at residential scale is presented next. The apparatus of the presented case study yields configurations for the programmable facade of a prototype house in Trento, through the management of variable transmittance materials by autonomous control. The dynamic, south facade of the prototype operates as a reconfigurable filter between exterior and interior. It filters solar radiation and heat by altering the chromatism and light transmittance of each individual windowpane. Varying the number and the distribution of the active windowpanes on the facade permits the regulation of the incoming sunlight and heat, and affects the building performance and aesthetics. 


\section{Background}

The prototype house in Trento, Italy is the result of the integration of innovative physical and computing architecture, passive and active building technologies. The house systems operate in autonomous, responsive and interactive modes interchangeably based on the conditions. The south-facing elevation of the prototype comprises one of the most significant house systems. Triple glazed, operable windows incorporating layers of electroactive materials permit the selective adjustment of natural light, heat and air. The programmable windows of the facade are managed by a model-based distributed control system that aims to reduce the use of the HVAC and artificial lighting, in order to keep the long-term energy consumption minimal.

The control system of the house operates in a consistent way to exploit the energy of the sun and the passive, thermal conservation capacity of the building envelope. During the hot summer days, protecting the interior from direct sun exposure to keep the interior temperature lower than the exterior becomes a high priority. Hence, the windowpanes are automatically set to their minimum solar transmittance value to effectively block the sun's heat. Conversely, during the cold winter days, exposing the interior to the warmth of the winter sun becomes a priority. Hence, the windowpanes are set to their maximum solar transmittance value to permit the storage of heat in the home's passive envelope. Generally, sunlight, heat, shade and fresh air are used to maintain comfortable conditions while keeping non-renewable energy expenditure minimal. The autonomous control system of the house maintains stability of indoor conditions whenever the building is occupied. The residents can specify their desired range of indoor comfort, or allow the system to take charge. Where uncertainty in weather and occupancy patterns poses a risk of failure, the control limits this risk by explicitly addressing uncertain factors.

After an overview of related background research, this presentation is divided in two parts, namely variable transmittance materials and model-based autonomous control. The first part exposes the electrochemical properties of the materials used in the dynamic facade. The second part presents the features of the model-based autonomous control system. The presentation concludes with a discussion summarizing the visions and contributions of this project.

The related work, a number of papers describe the state of the art in variable transmission materials, like electrochromic glass: Lee et al. [1] present a study in which the effects of electrochromic technology are monitored in a cube $3.0 \mathrm{~m} \times 3.0 \mathrm{~m} \times 3.0 \mathrm{~m}$; Hausler et al. [2] present a technical comparison of data determining the physical features of electrochromic glass; Selkowitz et al. [3] offer an overview on automated lighting and energy control systems; and finally, Mardaljevic et al. [4] review the historical basis of building compliance methods determining minimum energy efficiency and comfort standards and propose a new basis for more efficient metrics.

The application of AI methods to building control has been pursued by computational sustainability research: Mady et al. [5] are using the SMPC (stochastic model-predictive control) approach to significantly reduce the energy consumption of a building with stochastic occupancy model; Kolter et al. [6] model end-user energy consumption in residential and commercial buildings; $\mathrm{Li}$ et al. [7] use a robust plan executive for autonomous underwater vehicles. The proposed plan executive, p-Sulu (probabilistic Sulu), is built upon the iterative risk allocation algorithm [8] and a deterministic plan executive, Sulu [9].

\section{Variable Transmittance Materials}

From an energy conservation point of view, windows are a standard source of problems, by either admitting too much sunlight and heat when they are unwanted, or by blocking the warming sun when it is needed, and by providing unwanted thermal bridges to 
the outside. These inefficiencies often make the use of the HVAC system compulsory. The proposed dynamic windows use layers of variable transmittance materials holding the promise for energy-saving windows that respond dynamically to the amount of incident solar irradiance (Figs. 2 and 3). The south facade of the prototype house is a matrix of dynamic windows, also incorporating mechanical actuation and control capabilities applied on each window independently. This dynamic facade achieves three complementary objectives: (1) regulates the percentage of sun-heat penetrating the house; (2) regulates the interior daylight illuminance; and (3) regulates the pattern of the incoming airflow. The next
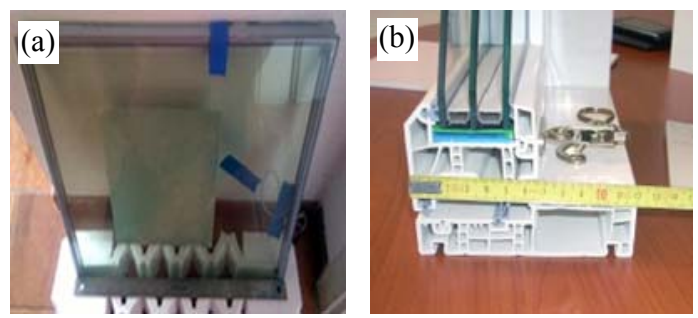

Fig. 2 Sage electrochromic glass pocket: (a) before; (b) after installation in the frame.

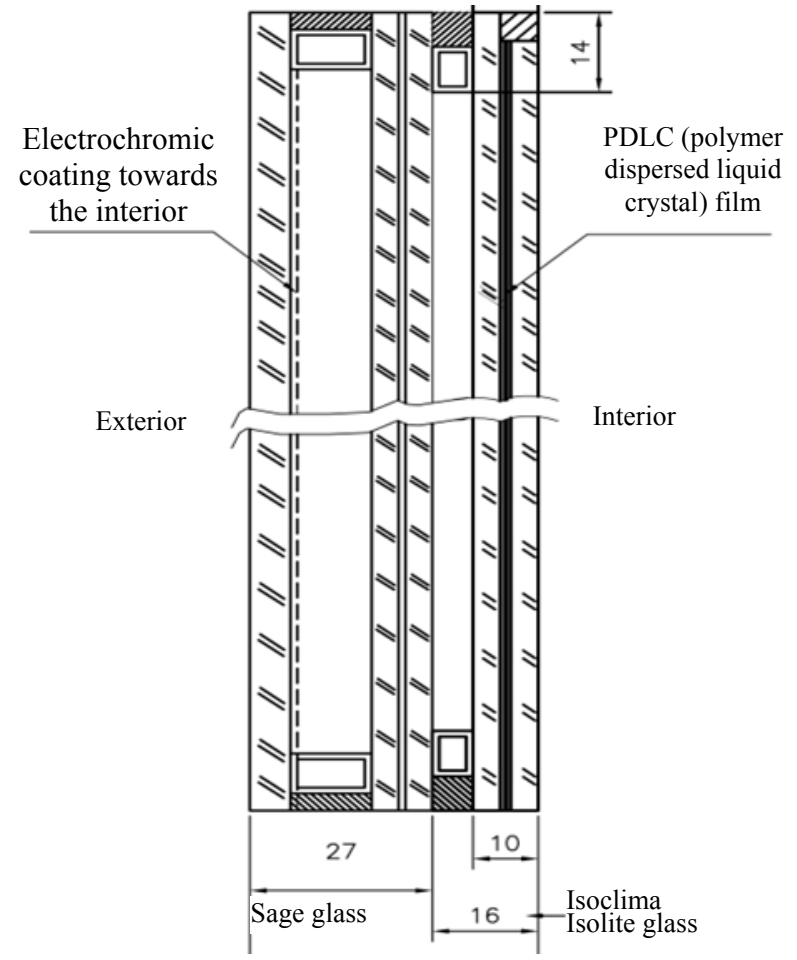

Fig. 3 Section of the triple glazed windowpane. two sections address the issues of sunlight and heat management by variable transmittance materials.

\subsection{Windows with Variable Transmittance Materials}

The south facade of the prototype operates as a programmable solar wall forming a $3 \times 9$ matrix of windows $0.700 \mathrm{~mm} \times 0.700 \mathrm{~mm}$ each. Each windowpane is triple glazed, $43 \mathrm{~mm}$ in thickness, and involves an overlay of two electroactive materials, namely an electrochromic coating and a PDLC (polymer dispersed liquid crystal) film. The first exterior glazing is a $6 \mathrm{~mm}$ in thickness SageGlass ${ }^{\circledR}$ tempered pane, on which an electrochromic coating is applied. This is followed by a $12 \mathrm{~mm}$ argon filled gap and a second $6 \mathrm{~mm}$ in thickness clear tempered pane. An argon-filled gap of $6 \mathrm{~mm}$ follows, and then the third interior glazing is a $10 \mathrm{~mm}$ in thickness glass-pack, involving two Isoclima-Isolite clear panes with a PDLC film applied in between (Fig. 3).

The electrochromic glass is a variable transmission material that provides precise adjustment of the incoming daylight and solar radiation. As a result, the windows have an adjustable SHGC (solar heat gain coefficient) ranging from 0.45 in their idle state to 0.10 in their obscured state. The modification of the glass state requires application of a low voltage current that is applied only during the transition time from one state to another. The power consumption ranges from $0.4 \mathrm{~W} / \mathrm{m}^{2}$ (average) to $2.5 \mathrm{~W} / \mathrm{m}^{2}$. The $U$ factor value indicating insulating performance (lower $U$ factor indicates higher insulating performance) is for the SAGE glass particularly satisfying, ranging from 0.29 for idle glass to 0.28 for active obscured glass. The transmittance value $(\tau)$ to the visible light for the particular type of SAGE electrochromic glass varies from $62 \%$ for idle glass to $3.5 \%$ for active obscured glass without ever compromising visibility. The above properties are highly desirable compared to those of conventional glazed windows. However, electrochromic windows are characterized by slow transition speeds, on the order of minutes, between the 
idle and obscured states. The transition time of the SAGE electrochromic glass is approximately $8 \mathrm{~min}$.

The ability of the PDLC film to modify its state from opaque to transparent allows for applications that secure privacy and provide the desirable degree of visibility. The PDLC film effectively blocks visibility and eliminates the need for mechanical parts like shutters or blinds. Hence, optical dimming and density variation replace the need for mechanical actuation with solid-state shading and glare. The standard state of the PDLC film is opaque. Unlike the electrochromic glass, the modification of the PDLC film requires constant application of an alternating electric field to remain in a transparency state. The power consumption is $<10 \mathrm{~W} / \mathrm{m}^{2}$. The $U$ factor value indicating the insulating performance of the PDLC film is insignificant $\left(U_{g}=0.7\right)$. The transmittance value $(\tau)$ to the visible light is $63 \%$ for the PDLC film when the film is in its transparent state and zero when the film switches to its opaque state. Lastly, PDLC is characterized by fast transition speeds, on the order of seconds, between opaque and transparent states.

The results from the activation of the electroactive materials is presented in Fig. 4: (a) The electrochromic layer is inactive and the PDLC layer is active; (b) Both the electrochromic and PDLC layers are active; (c) The electrochromic layer is active and the PDLC layer is inactive; and (d) Both the electrochromic and the PDLC layers are inactive.

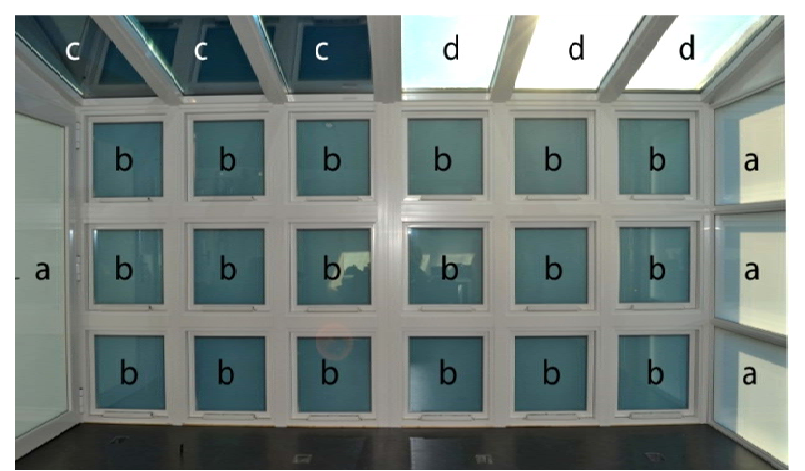

Fig. 4 Activation of materials: (a) electrochromic layer inactive and PDLC layer active; (b) electrochromic and PDLC layers active; (c) electrochromic layer active and PDLC layer inactive; (d) electrochromic and PDLC layers inactive.
Altering the states of the windows in real-time is not a typical application of the electrochromic or PDLC products. It is a custom feature developed in the laboratory. At night, all the windowpanes are switched by default to their opaque state and inhabitants are given full control. During the day, the windows are adjusted counteractively based on the environmental management priorities. At all times, the windows are directed in a concerted manner by the high-level control without interfering with each other. However, at a local level, each window is driven by its own low-level control and custom electronics that enable its activation. Since the response times, and the optical, thermal and power consumption attributes of the electrochromic and PDLC materials vary, their activation processing requires precise coordination. The SAGE system provides the capability to address individual panels and control their tint level within a four-valued scale $0 \%, 6 \%, 20 \%$ or $100 \%$. The PDLC film also permits variable tinting based on input from sensors. The on/off controlling of each windowpane happens through a low-level controller, a SAGE-Bus. The communication of the SAGE low-level controllers with the high-level control is achieved through RS232 serial ports. Each window provides feedback to the high-level control that activates the required parts of the facade. A distributed control network including one master node and up to 122 slave nodes is employed for this purpose. Each slave node involves a motor driver, a relay circuit, a sensor unit and a communication module. The microprocessor on the slave node processes the incoming commands sent from the master node and transmits the control signal to the function modules. An accelerometer connected to the slave node takes real-time measurement of the window angles. A motor driver compares the desired and measured angles and controls the opening angles with $\pm 2.5^{\circ}$ of accuracy. The relay circuit turns the PLDC film on or off, while the SAGE system integration module controls the tint state of each windowpane (Fig. 5). 


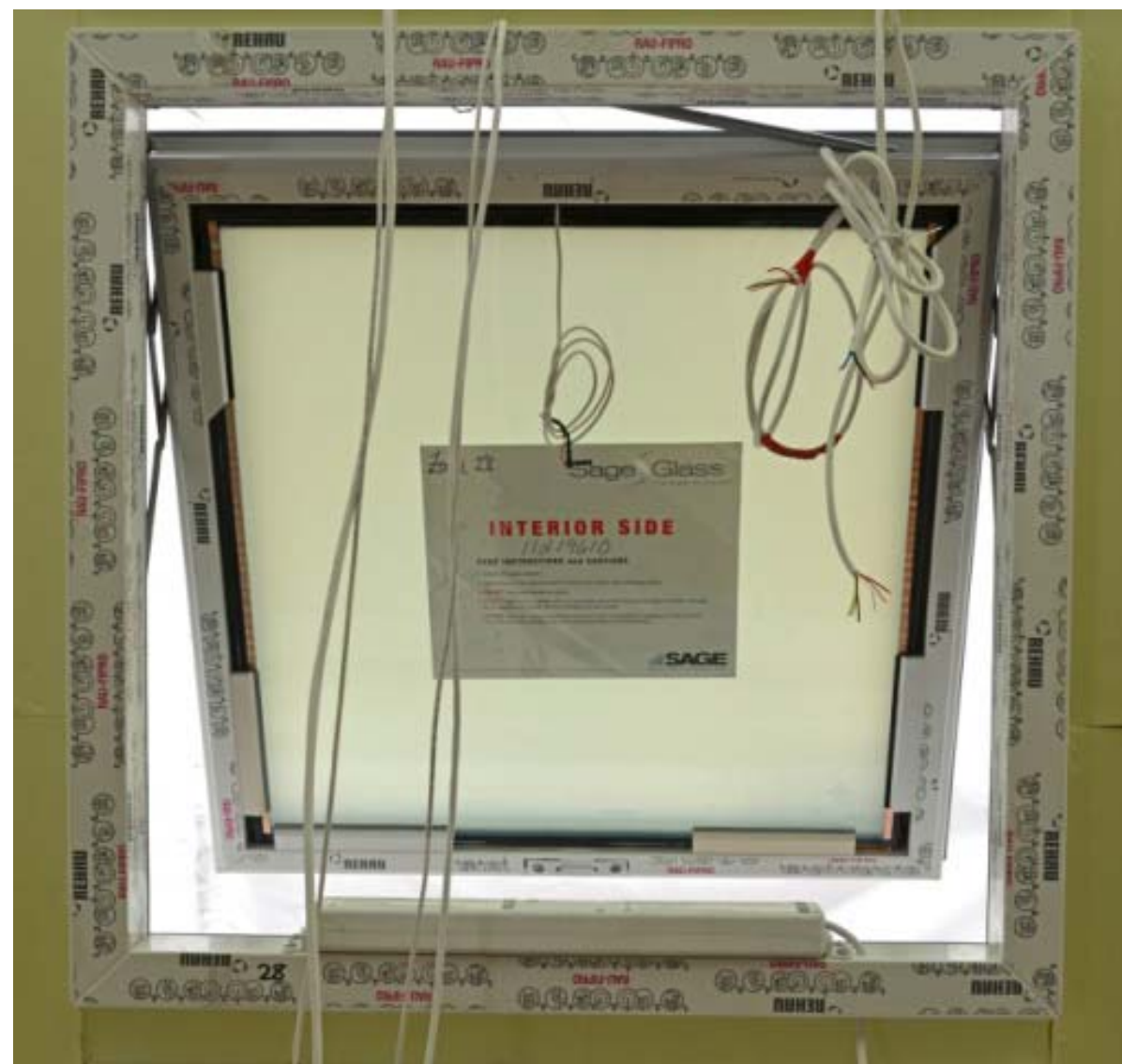

Fig. 5 Window unit during installation, visible are the electrochromic layer in clear state, the PDLC layer in opaque state, the motor driver and the necessary wiring for their operation.

\subsection{Daylight Simulation}

Preliminary simulation analysis of data on the performance of the electrochromic and PDLC materials made possible the extraction of useful information on how their activation affects the amount of the incoming solar heat. The fraction of the solar irradiance incident that is admitted through a windowpane is determined by the SHGC. The SHGC of a material is the sum of the transmittance and the fraction of the absorption that is eventually transmitted. Given a window area $A$ and incident solar irradiance $I(t)$, the rate of heat transfer $q(t)$ into the interior space is:

$$
q(t)=S H G C \cdot A \cdot I(t)
$$

Hence, the SHGC of the fenestration is a function of the incident solar irradiance. Fig. 6 presents plots of this function for traditional double-glazed windows with venetian blinds, in comparison to triple-gazed, argon filled dynamic windows. More specifically SHGC versus solar irradiance diagrams are presented for: (a) venetian blinds in winter; (b) PDLC active layer in winter; (c) electrochromic layer in winter; (d) venetian blinds in summer; (e) PDLC active layer in summer; (f) electrochromic layer in summer. These datum indicate the major role of the electrochromic layer in controlling the heat transfer.

Analysis and evaluation of the performance of the electrochromic glass allowed the extraction of a general predictive model on how its activation affects the level of thermal comfort and interior daylight illuminance on any time interval throughout the four seasons. The simulation tests were performed on a digital reproduction of the house. The simulation software used in the illuminance analysis was Relux professional by Relux Informatik AG, while Relux vision (a plug-in of Relux professional) was used in 


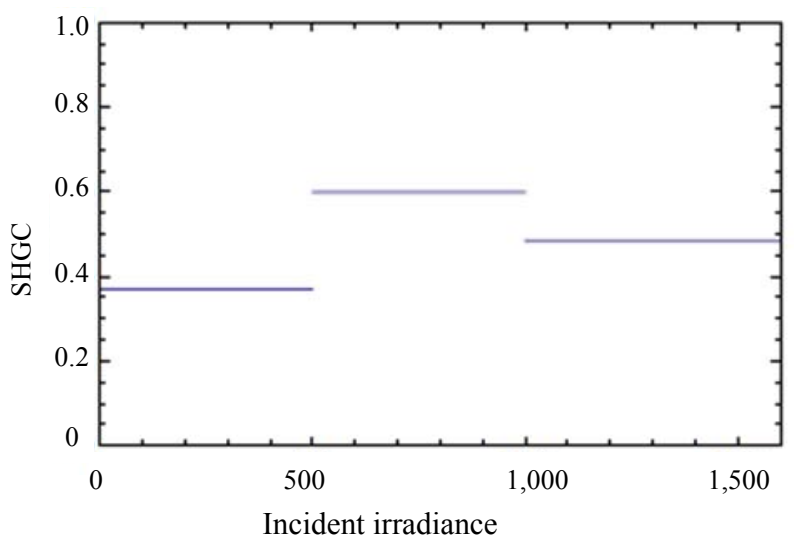

(a) Venetian blinds-winter

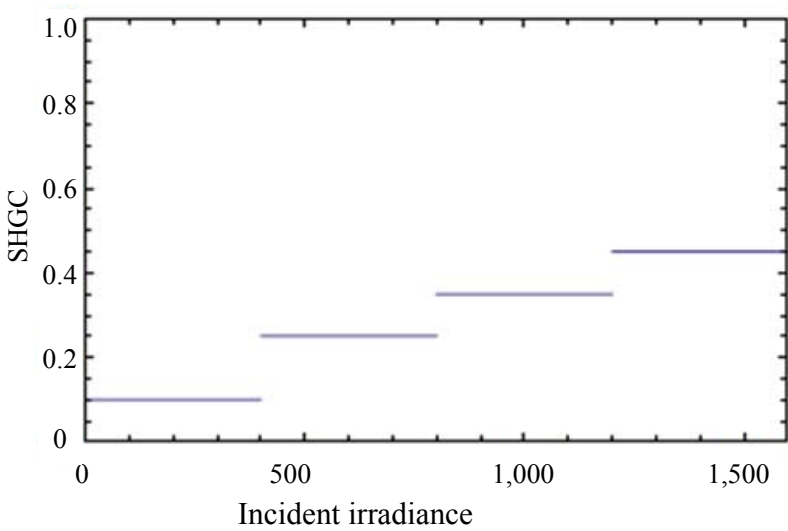

(c) Electrochromic windows - winter

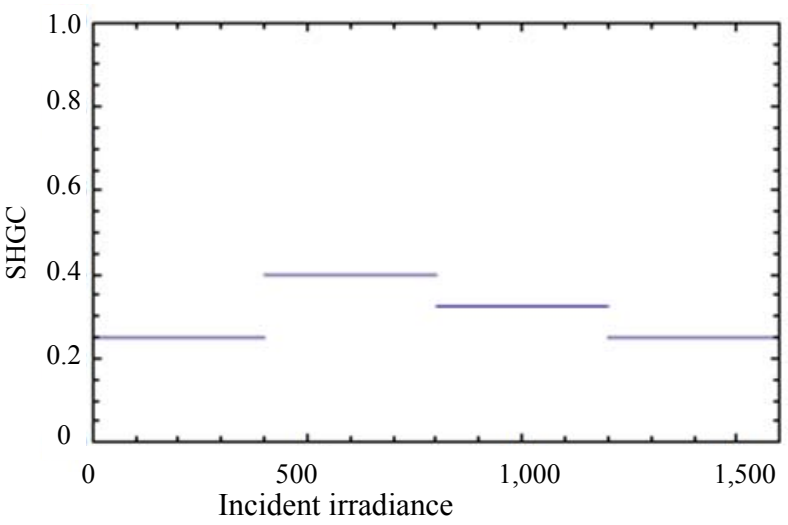

(e) PDLC windows - summer

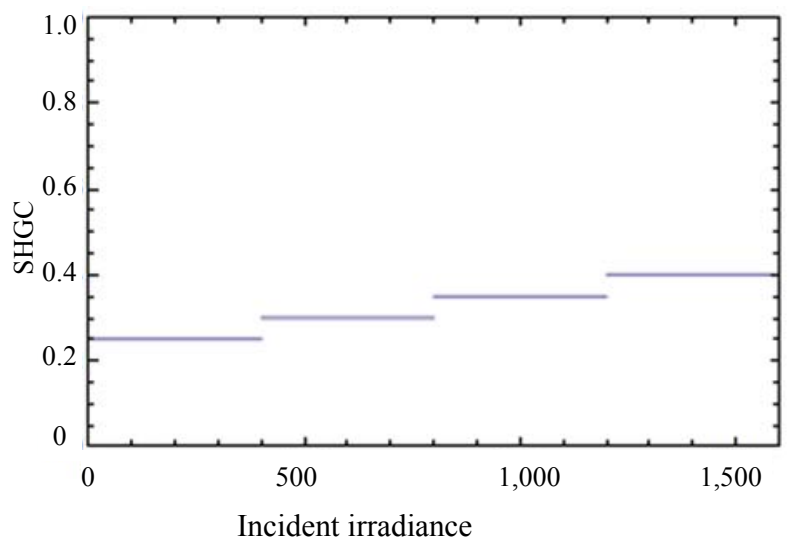

(b) PDLC windows - winter

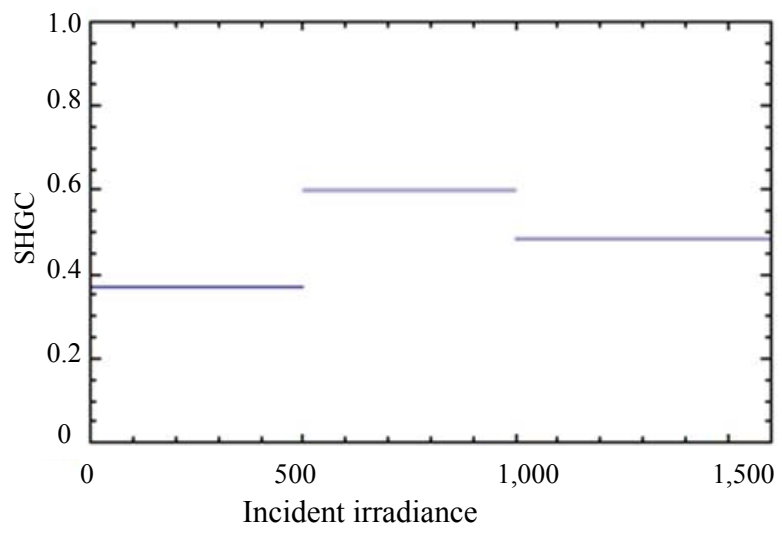

(d) Venetian blinds - summer

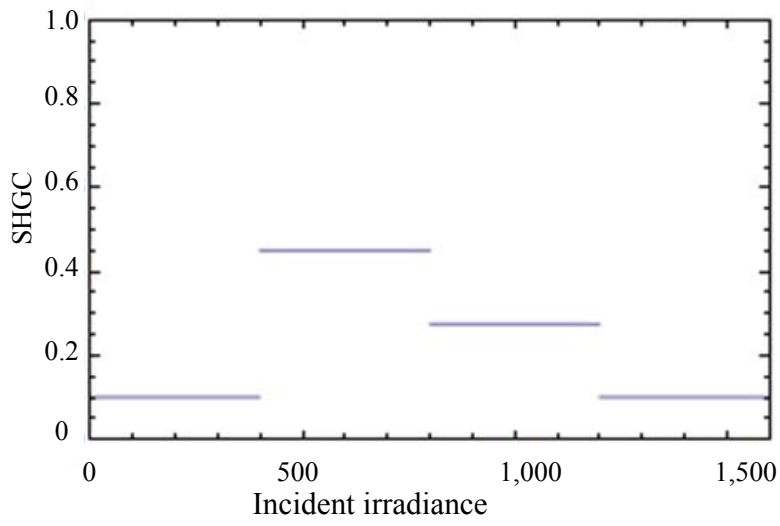

(f) Electrochromic windows - summer

Fig. 6 SHGC versus solar irradiance: (a) venetian blinds in winter; (b) PDLC active layer in winter; (c) electrochromic layer in winter; (d) venetian blinds in summer; (e) PDLC active layer in summer; (f) electrochromic layer in summer.

the ray-tracing simulation. Climatic data for the location of the prototype, in Trento, Northern Italy, was provided by the database of the software, which also integrates the lighting standards determined by Italian law. SAGE Electrochromics provided specifications for the electrochromic material used on the facade.

Two models - established by the CIE (Commission Internationale de 1' Eclairage)—were used in the analysis and evaluation of natural light. The first was 
the standard overcast sky model, which provides an account of the sunlight emitted through cloudy sky. The second was the standard clear sky model, which computes sunlight under the assumption that the sun is the single lighting source, without calculating the diffused and reflected light by the sky.

Typical outputs of the simulations included the minimum, maximum and average values of illuminance, the uniformity and average daylight factor values, the isolux maps for assigned planes, and the tri-dimensional illuminance diagrams. Typically, the daylight conditions of an interior space are captured by the average illumination $E_{\text {ave }}$, the uniformity $G_{l}$ and the daylight factor $D_{a v}$. Accordingly, the minimum daytime value of illuminance $E_{\min }$ was set to 500 lux. The uniformity value $G_{l}$ that captures the smoothness of daylight distribution, defined by the ratio $E_{\text {min }} / E_{\text {ave }}$, was set to $G_{l}=0.5$. Lastly, the daylight factor $D_{a v}$ representing a physical constant for all windows was set to $D_{a v}=3$.

The simulation tests allowed identifying the required number $x$ of active windows to reach the value of 500 lux for every day of the year. This made possible the extraction of a general predictive model associating coverage ratio $\alpha$, which corresponds to the ratio of the number $x$ of active windows versus the total number of windows, and illuminance values in any specified day and time interval during the year. Based on this model the number of active electrochromic windows ensuring the illuminance threshold of 500 lux in overcast sky ranges from $1 / 2$ to $3 / 4$ of the total number of windows. For a total number of 100 windows the required number $x$ (Fig. 7) of active electrochromic windowpanes will range from 50 to 75 . A table and the corresponding diagram with the results ensuring 500 lux for the 12 months of the year, at 1 p.m. in Trento, Northern Italy, and standard overcast sky appear in Table 1 and Fig. 7.

Table 1 The results ensuring 500 lux for the 12 months of the year, at 1 p.m. in Trento, Northern Italy.

\begin{tabular}{llrlclc}
\hline No. & $\begin{array}{l}\text { 1 p.m. on } \\
\text { 21st day }\end{array}$ & $x$ & $\alpha$ & No. & 1 p.m. on 21st day & $x$ \\
\hline 1 & December & 0 & 0.00 & 7 & June & 75 \\
2 & January & 18 & 0.18 & 8 & July & 75 \\
3 & February & 45 & 0.45 & 9 & August & 72 \\
4 & March & 62 & 0.62 & 10 & September & 63 \\
5 & April & 72 & 0.72 & 11 & October & 0.75 \\
6 & May & 75 & 0.75 & 12 & November & 0.63 \\
\hline
\end{tabular}

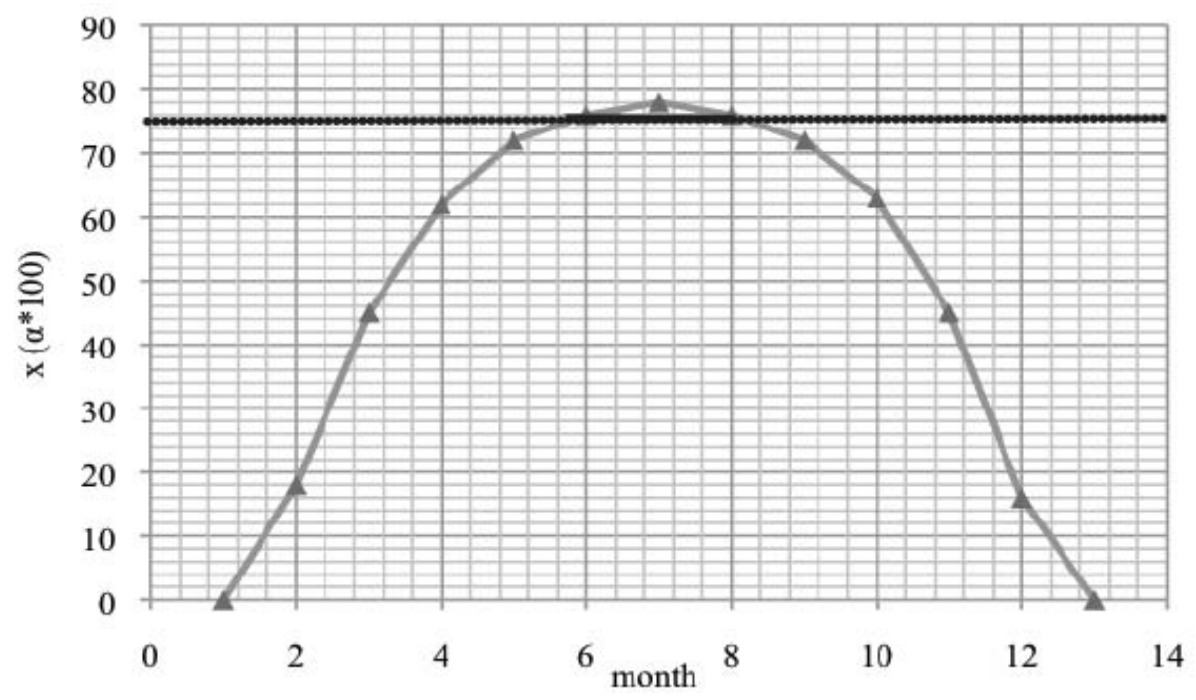

Fig. 7 The maximum numbers of active electrochromic windowpanes $x$ throughout the year ensuring 500 lux in the prototype at 1 p.m. and standard overcast sky. 
The produced database was used as foundation for optimizing the performance of the facade. The combination of this database with real time feedback from sensors enables the dynamic reprogramming of the electrochromic material. The overall apparatus achieves two complementary objectives:

(1) Determines the required optimum number of active electrochromic glass units to reach the desired interior temperature and illuminance;

(2) Confines any electrochromic facade pattern to this threshold.

More importantly, the simulations confirmed that the electrochromic material could switch from one facade pattern to another while maintaining the same activation ratio, in order to possibly satisfy other factors. Additional details on the simulation process of the electrochromic windows and the development of a generative grammar capturing the pattern language of the optimum facade patterns can be found in Ref. [10].

\section{Model-Based Autonomous Control}

Modern fenestration systems involving curtain walls, blinds, shutters or screens admit selectively natural elements to building interiors. Traditional environmental management techniques rely on reactive control and on the extensive use of the HVAC system to maintain desired conditions in air-sealed interiors. In this way, the goal of energy efficiency is compromised. In 2010, residential buildings consumed $21.52 \%$ of the total energy usage in the US [11] with heating and cooling being responsible for the $38.2 \%$ of the residential energy consumption. It is projected that energy savings of up to $35 \%$ can be achieved by simply optimizing the use of the HVAC system in more effective ways [12].

The prototype house in Trento offers an alternative environmental management scheme involving variable transmittance windows and autonomous control, the objective being to significantly reduce energy consumption and to enhance comfort. Next it is presented the model-based autonomous control apparatus of the house. The control system manages the interior atmosphere of the prototype, adjusts the state of the dynamic facade and limits the necessity for HVAC use while keeping the long-term energy consumption minimum. The three technical challenges enabling the achievement of autonomous control are outlined and the required inputs-outputs as well as the algorithm of the control system are explained.

\subsection{Autonomous Control Technical Challenges}

Three words summarize the technical challenges enabling the achievement of autonomous control at the prototype, namely comfort, sustainability and convenience. Comfort is achieved by allowing the flexible setting of the user's preferences into the system. Under uncertain weather conditions the system provides a probabilistic guarantee that the desired comfort levels will be maintained. Sustainability is achieved by effectively minimizing the expenditure of non-renewable energy sources. And convenience is ascertained by minimizing the user's effort in the achievement of the two previous goals.

Firstly, the residential character of the prototype makes it essential that the control system does not simply minimize energy use, but also flexibly adapts energy management to the activities of the residents. Most of the existing environmental management systems do not acknowledge this possibility. Standard HVAC systems receive a single set point as input and operate reactively to maintain it. More sophisticated controllers allow the residents to input multiple set points. Deterministic systems like these can not accommodate the full spectrum of a resident's needs. The autonomous control of the prototype provides the capacity to represent a detailed schedule and desired ranges of comfort (i.e., state constraints in a continuous domain), and the ability to plan over these. This capability is called [13] goal-directed planning with continuous effect and the language used to represent a detailed schedule with constraints is called 
a CCQSP (chance-constrained qualitative state plan).

Secondly, the performance philosophy guiding the design of the prototype is that the house must consume as little non-renewable energy as possible. Parallel management of the HVAC system, of the variable transmittance windows, and the thermal conservation capabilities of the house envelope can have vast contribution in achieving this objective. To make the goal of distributed control possible the control system must receive data capturing the dynamics of the house environment, and it must calculate a control plan that minimizes use of the HVAC and of non-renewable energy sources. In technical terms, the controller must achieve optimal planning of the systems [14].

Thirdly, environmental management involves a risk of failing to maintain interior comfort within some specified range due to unexpected changes. Weather patterns including temperature and solar radiation are uncertain due to the unpredictability of the atmosphere. Weather forecasts are inconsistent, and the same is true for the preferences of the inhabitants. A controller operating in a stochastic environment needs to account for uncertainty while attempting to plan optimally in order to avoid constraint violations. A probabilistic guarantee that the resident's comfort constraints will not be violated, can be provided by explicitly acknowledging the sources of uncertainty and planning accordingly. An approach of this kind is referenced as risk-sensitive planning [14].

To address the above technical challenges, namely risk-sensitive, optimal, goal-directed planning with continuous effect, an on-line risk-sensitive planner called p-Sulu (probabilistic Sulu) is used [15]. P-Sulu leverages an IRA (iterative risk allocation) algorithm to provide robust planning in the context of environmental management. It also expands upon previous work [16] to extend the on-line planning to include executive capabilities that perform receding horizon control.

\subsection{Probabilistic Sulu}

Previous research [15] describes a risk-sensitive finite-horizon planner called Sulu. Sulu partially meets the three technical challenges of autonomous control for the house. However, Sulu is an off-line planner, meaning that it pre-plans the control sequence for the entire plan duration of a task. Hence, it can not possibly be used for the task of managing the prototype due to two problems. First, a house is operated continuously with no interruptions, while Sulu can plan only for finite time duration. Second, efficient environmental control requires frequent re-planning every few seconds, while Sulu's solution time is on the order of minutes. For example, a simple path-planning problem with 10 time steps requires $30 \mathrm{~s}$ to solve [15].

The newly developed $\mathrm{p}$-Sulu is an on-line risk-sensitive planner that extends Sulu by providing solutions to the abovementioned problems. To overcome the first problem a receding horizon control approach is adopted. At each planning cycle, a planning problem is solved with a finite duration, which is called a horizon. In the next planning cycle, the planning problem is solved again over a horizon with the same duration starting from the current time (hence, the horizon is "receding") by considering the latest observation of uncertain parameters. This re-planning process is repeated with a fixed time interval. The second problem is solved by building upon an anytime algorithm for chance-constrained programming called IRA (iterative risk allocation) [8]. IRA was originally developed for path planning problems without time-evolved goals. The proposed new approach extends IRA to deal with time-evolved goals [16].

P-Sulu takes as input a representation called a CCQSP (chance-constrained qualitative state plan). CCQSP encodes the schedule and comfort preferences of the residents as well as the acceptable level of risking a constraint violation and failing to maintain 
the desired conditions. The time schedule and comfort preferences of the residents are encoded as time-evolved goals. Time-evolved goals are constraints placed on the state of the system jointly with temporal information describing their timeframe. Examples of time-evolved goals might include "maintain a sleep temperature until waking-up time", or "maintain room temperature until sleeping time". The level of risk is encoded by chance constraints that bind the probability of a constraint violation. For example, chance constraints may specify that the level of risk has a $1 \%$ upper bound on the probability of human discomfort and a $0.01 \%$ upper bound on the probability of letting the house equipment freeze. Given a CCQSP, the p-Sulu controller has to find a control sequence that satisfies all time-evolved goals within the failure probability specified by the chance constraints.

P-Sulu explicitly takes into account a stochastic plan model, which specifies probabilistic state transitions in a continuous domain [13]. The plan executive p-Sulu takes as input a linear model to describe the system being controlled. The model is of the form:

$$
x_{t+1}=A_{t} x_{t}+B_{t} u_{t}+w_{t}
$$

where, $x_{t}$ is a continuous state vector at time $t, u_{t}$ is a continuous control vector at $t$, and $w_{t}$ is a disturbance whose probability distribution is known.
A CCQSP may be depicted as an acyclic directed graph. An example of this input is shown in Fig. 8.

The schedule of Fig. 8 is described in English, as follows:

"Maintain a comfortable sleeping temperature until waking-up time. Then, maintain room temperature until it is time to go to work. I may work at home, but I have to do $5 \mathrm{~h}$ of work at the office sometime between 9 a.m. and 6 p.m.. No temperature constraints while I am away. When I get home, maintain room temperature until bedtime. The probability of failure of these episodes must be less than $1 \%$. The entire time the house must not get so cold that the pipes freeze. Limit the probability of such a failure to $0.01 \%$

A CCQSP is a quadruple $P=(C, A, T, C)$, where, $C$ is a set of discrete events, $A$ is a set of episodes, $T$ is a set of temporal constraints, and $C$ is a set of chance constraints. An event, illustrated as a circle in the graph of Fig. 8, represents a point in time, to which an execution time is assigned. An episode, depicted as a rectangle in the graph of Fig. 8, specifies the desired state of the system under control over a time interval. It is a triple $a=\left\langle e_{a}^{S}, e^{E}{ }_{a}, R_{a}\right\rangle$, where, $e_{a}^{S}$ and $e^{E}{ }_{a}$ are the start and end events of the episode, respectively. Each episode $a$ has a feasible state region $R_{a}$. For example, $R_{a}$ for "maintain sleep temperature" is a closed interval on the indoor temperature $\left(18-22^{\circ} \mathrm{C}\right)$. A

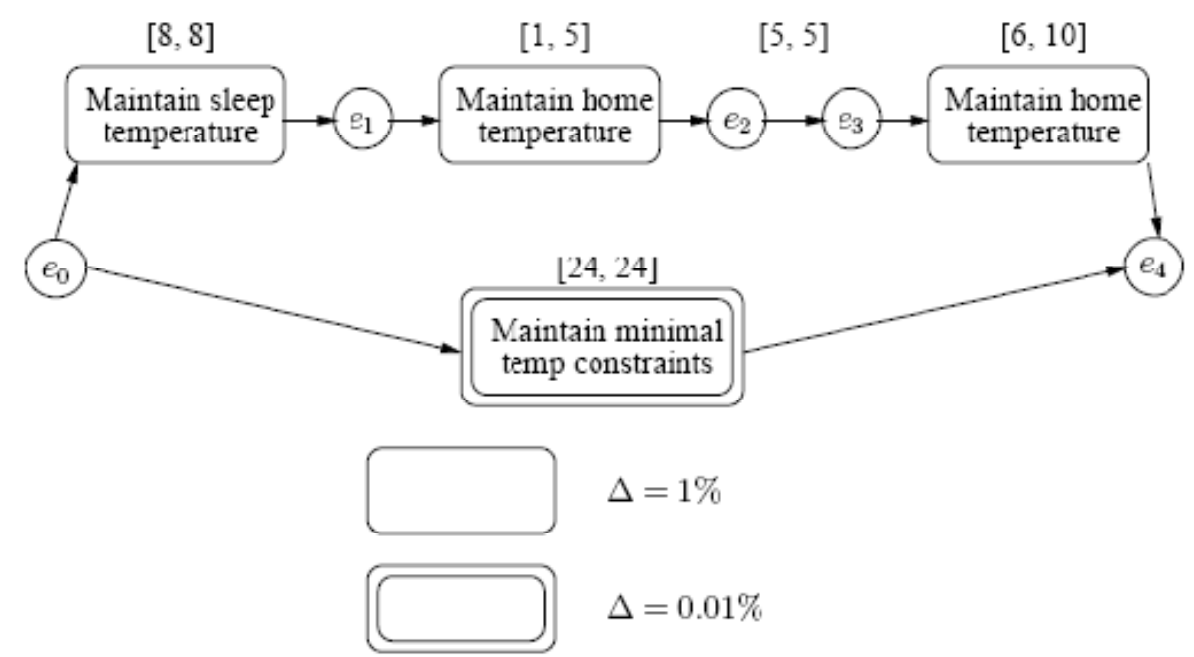

Fig. 8 A CCQSP illustrated by an Acylic directed graph depicting the resident's schedule in a planning problem. 
flexible temporal constraint is denoted by two numbers in a bracket $\left(l b_{e}^{e^{\prime}}, u b_{e}^{e^{\prime}}\right)$ that specify an upper bound $u b_{e}^{e^{\prime}}$ and a lower bound $l b_{e}^{e^{\prime}}$ on the temporal distance between two discrete events $e$, $e^{\prime}$ within the set $C$ of all discrete events $\left(e, e^{\prime} \in \epsilon\right)$. Last, a chance constraint specifies a lower bound on the probability of failing to satisfy a set of episodes during execution. It is a pair $c=\left\langle\Delta_{c}, \Psi_{c}\right\rangle$, where, $\Delta_{c}$ is the risk bound and $\Psi_{c}$ is the set of episodes associated with a chance constraint $c$. In the CCQSP of Fig. 8, the first chance constraint $\Delta=1 \%$ is associated with three episodes, namely an initial "maintain sleep temperature" episode and two "maintain home temperature" episodes.

P-Sulu has two outputs. The first output is an executable sequence $u_{0} \ldots u_{T}$ that minimizes a given cost function and satisfies all temporal and dynamic constraints. These are specified by the input CCQSP based on the probability determined by the chance constraints. In the prototype the outputs include the state of the windows as well as the heat output of the HVAC system. Minimizing energy consumption is possible over finite horizons, but the executive must extend over an infinite time-horizon. Hence, the controller provides an optimal plan over consecutive finite planning horizons. The second output of $\mathrm{p}$-Sulu is an optimal schedule. Let $s_{e} \in I R_{+}$be an execution time of the event $e$, with $I R_{+}$a set of non-negative real numbers. A schedule is the assignment of execution times $s_{e}$ to all events $e$ in the set $C$ of discrete events of the input CCQSP. Flexibility in the temporal constraints allows flexibility in the time in which each episode can be executed. If all temporal constraints are fixed, then the execution times are also fixed and the schedule is non-flexible.

Overall, the P-Sulu controller consists of two nested loops, an outer and an inner. The outer loop performs continuous receding horizon planning through consecutive calls to a stochastic CCQSP planner. The CCQSP planning is implemented by the inner loop through the IRA-CCQSP algorithm, which performs a series of fixed-risk planning steps. An overview of the IRA-CCQSP algorithm is presented in the next example. A room temperature control problem with a 24-hour planning horizon is depicted in the next Fig. 9. A fixed schedule is assumed. The resident wakes up at 8 a.m. leaves home at 12 p.m. returns to home at 5 p.m. and sleeps at 12 a.m.. The room temperature is required to be within specific ranges. Previous research [8] shows that in order to optimally solve a joint chance constrained optimization problem, it is essential to optimize the allocation of risk to each constraint. The IRA-CCQSP algorithm optimizes risk allocation for a CCQSP planning problem. It satisfies chance constraints by setting a safety margin (shadowed areas in Fig. 9) along the boundaries of the

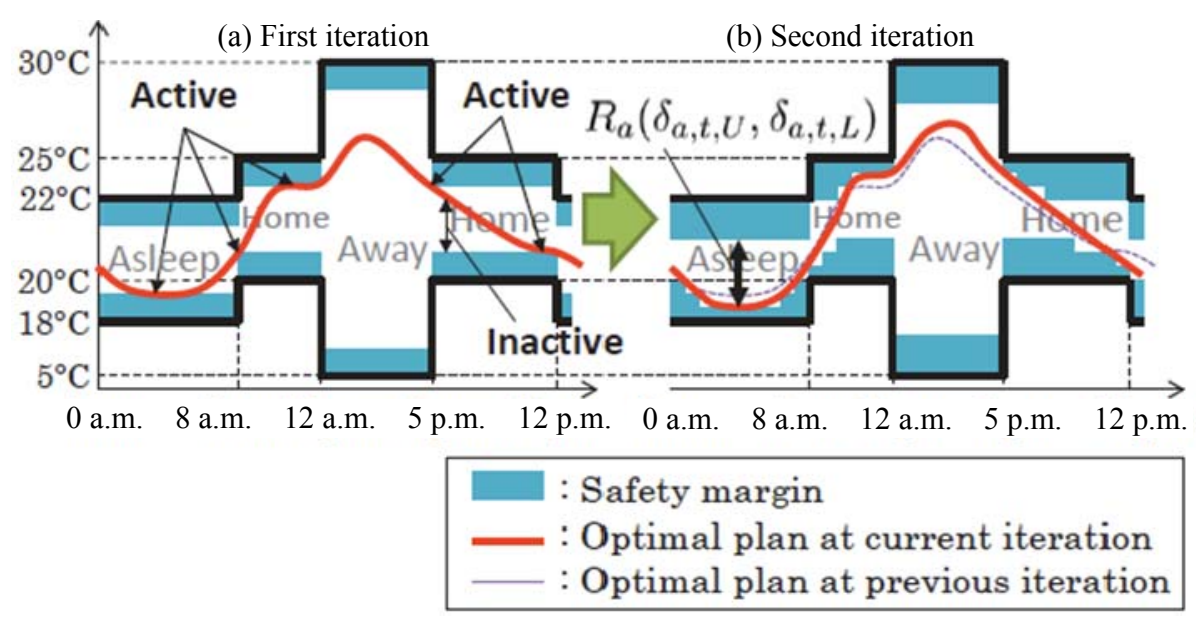

Fig. 9 Overview of the iterative risk allocation algorithm. 
constraints and by planning a nominal state trajectory to remain outside of the safety margin. The width of this margin is determined in a way that the probability of constraint violation is below the risk allocated to each constraint.

IRA-CCQSP is initialized with an arbitrary feasible risk allocation, such as a uniform one shown in Fig. 9a, and improves the risk allocation through iteration. A fixed-risk CCQSP planning problem is solved in successive iterations. A state trajectory is planned that minimizes cost and does not violate the safety margin. The plan minimizes energy consumption by lowering the temperature during the night and heating the room using the sun during the day. The plan heats the room to a maximum temperature before the sunset at around 5 p.m.. Thermal energy is stored in the massive envelope, so that the use of the HVAC system can be minimized during the night. Based on the plan of Fig. 9a the constraints are active at a few points in time, and inactive at all other points. To improve energy savings IRA removes the risk that was allocated to the inactive constraints and reallocates it to the active constraint. Reducing risk allocation results in a wider safety margin. Increasing risk allocation results in a narrower safety margin. The new risk allocation results in the safety margin are shown in Fig. 9b. IRA then solves the fixed-risk CCQSP planning problem again to obtain the optimal plan that does not violate the new safety margin. The new plan is more energy efficient than the one in the first iteration, since the temperature can be lower during the night and it is higher in the evening. In this way, the algorithm reallocates the risk again from inactive constraints to active constraints iteration after iteration. And the cost function value (i.e., energy consumption) decreases monotonically during successive iterations.

\subsection{P-Sulu Simulations}

The next simulation experiments demonstrate the two important capabilities of p-Sulu controller, namely optimal plan execution with flexible schedule and risk-sensitive plan execution with chance constraints. In the experiments, p-Sulu was implemented in $\mathrm{C}++$ and all trials were run on a machine with a Core i7 $2.67 \mathrm{GHz}$ processor and $8 \mathrm{~GB}$ of RAM. It was assumed that a resident can specify one of thee ranges: home, asleep and away. It was further assumed that the temperature must remain between $20^{\circ} \mathrm{C}$ and $25{ }^{\circ} \mathrm{C}$ while the resident is at home, between $18{ }^{\circ} \mathrm{C}$ and $22{ }^{\circ} \mathrm{C}$ while asleep, and above $5{ }^{\circ} \mathrm{C}$ while away, to ensure that the pipes will not freeze. Home and asleep episodes were associated with a single chance constraint class, with risk bound $1 \%$. This is the risk, the resident is willing to take that the temperature may become uncomfortable. Away episodes were associated to a single chance constraint class with risk bound $0.01 \%$. This is the acceptable risk that the pipes may freeze. A disturbance $w_{t}$ drawn from a Gaussian distribution with standard deviation $\sigma^{i n}{ }_{t}=0.5^{\circ} \mathrm{C}$ was also introduced.

First simulations of p-Sulu with a flexible and a fixed schedule are compared. For a flexible schedule, the temporal constraints represented by the CCQSP in Fig. 8 are set to remain flexible. For example, the residents will be out for work for $5 \mathrm{~h}$, but the time of their departure remains flexible between 9 a.m. and 1 p.m.. Conversely, for a fixed schedule, the residents always leave home at 9 a.m. and return at 2 p.m.. Table 2 presents a comparison of the results of the stochastic simulation for both the flexible and fixed weeklong scenarios in the winter (January 1), spring (April 1), summer (July 1), and autumn (October 1).

Fig. 10 shows the resulting nominal indoor

Table 1 Simulation of energy use on the prototype, using both fixed and flexible time constraints.

\begin{tabular}{lllll}
\hline & Winter & Summer & Spring & Autumn \\
\hline Flexible & $1.9379 \times 10^{4}$ & $3.4729 \times 10^{4}$ & $3.3707 \times 10^{4}$ & $3.8181 \times 10^{4}$ \\
Fixed & $2.1625 \times 10^{4}$ & $1.5295 \times 10^{4}$ & $3.4253 \times 10^{4}$ & $3.8445 \times 10^{4}$ \\
\hline
\end{tabular}


temperature in the winter, as well as the upper and lower bounds in room temperature corresponding to the optimal settings for flexible and fixed schedules. The optimal schedule is for the resident to leave home at $1 \mathrm{p} . \mathrm{m}$. and return at $6 \mathrm{p} . \mathrm{m}$. both in the winter and summer. This schedule optimization results in energy savings of $10.4 \%, 1.6 \%, 1.6 \%$ and $0.7 \%$ in the winter, spring, summer and autumn, respectively, compared to the fixed schedule approach. The performance of the fixed schedule approach varies by the schedule. If the given schedule happens to coincide with the optimal one, the resulting energy consumption is identical to the one with a flexible schedule. Since p-Sulu selects the best schedule among all the feasible ones, the resulting energy savings are always better than or equal to that of the fixed schedule approach.

The capability of p-Sulu in planning with chance constraints is demonstrated next. The performance of p-Sulu is evaluated on the basis of both energy savings and probability of constraint violations. Two additional baseline models are considered, for comparison: (1) a standard PID (proportional-integral-derivative) controller that attempts to minimize error based on a given set-point; (2) Sulu [9], a deterministic MPC (model predictive control) plan executive, which can handle flexible temporal constraints but can not consider chance constraints. Accordingly, first, p-Sulu is compared to

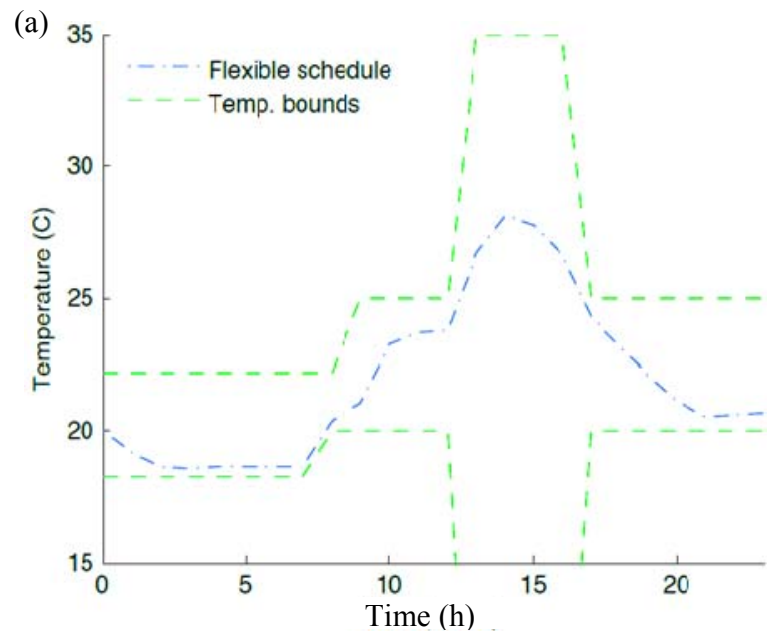

the PID to allow comparison of the energy savings of an MPC-based control and a traditional heating control. The set point of the PID controller was $21^{\circ} \mathrm{C}$, a point that is feasible in every state. Second, $\mathrm{p}$-Sulu is compared to Sulu to demonstrate the robustness to failure that arises from risk-sensitive control. By comparing p-Sulu with Sulu, it is possible to evaluate the benefit of introducing chance constraints. The simulation for each controller is based on a sample resident CCQSP that spans a week. Each controller is evaluated on the basis of energy use and percentage of failure due to constraint violations. Fig. 11 illustrates the results of a stochastic simulation over two different days of the year.

Sulu plans right to the edge of the constraints, often violating constraints when a disturbance is introduced, while p-Sulu respects a safety margin. Table 3 presents the results of the Monte-Carlo simulation on a weeklong scenario, averaged over 100 trials each with $\Delta=0.1$. The table shows that the MPC-based approaches (i.e., p-Sulu and Sulu) can significantly reduce energy consumption. P-Sulu yields energy savings of $51 \%$ over the PID controller during winter. During spring, summer and autumn, p-Sulu yields $15 \%, 17 \%$ and $4 \%$ energy savings, respectively. Sulu also achieves $58 \%, 22 \%$ and $8 \%$ energy savings compared to the PID controller during winter, spring and autumn, respectively.

(b)

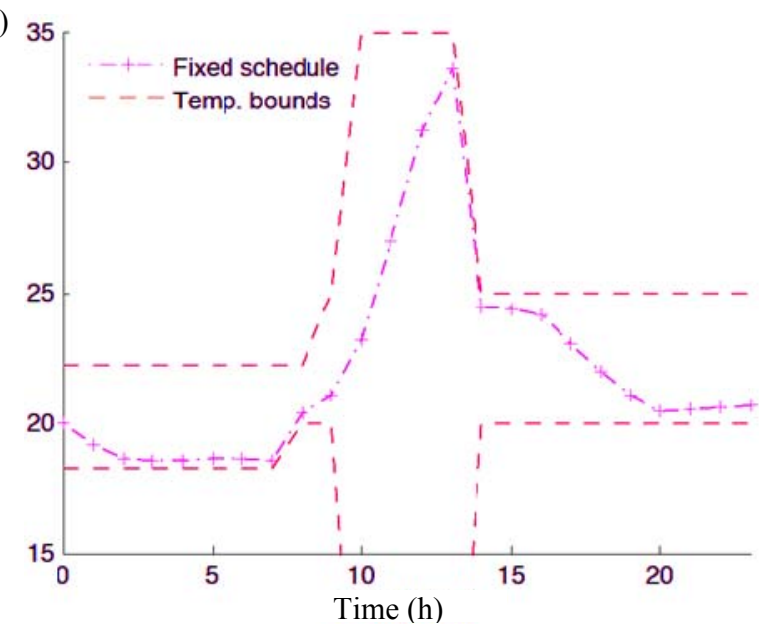

Fig. 10 Results of execution of p-Sulu control on Jan 1st: (a) with a flexible schedule; (b) with a fixed schedule. 

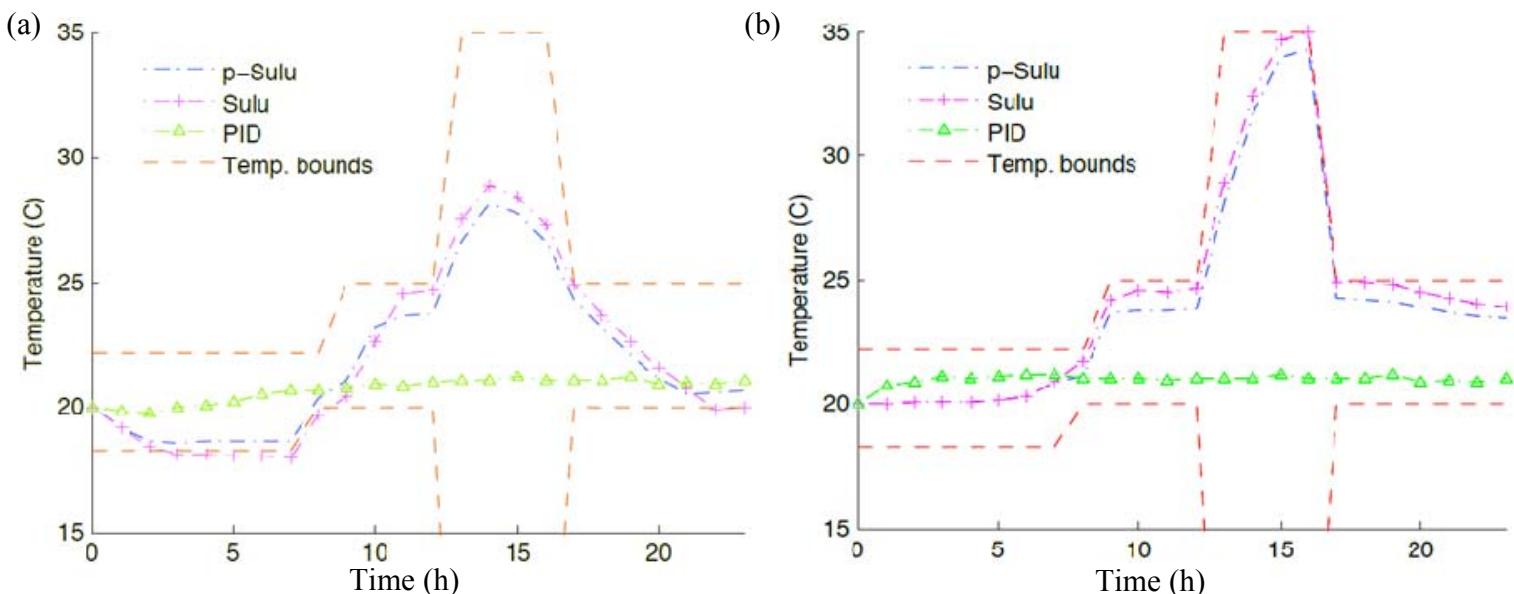

Fig. 11 Results for PID, Sulu and p-Sulu controllers on: (a) January 1; (b) July 1, p-Sulu controller appears in blue line.

Table 2 Comparison of energy use and failure rates of the p-Sulu, Sulu and PID controllers over a week long schedule in all four seasons.

\begin{tabular}{|c|c|c|c|c|c|c|c|c|}
\hline & \multicolumn{2}{|c|}{ Winter } & \multicolumn{2}{|c|}{ Summer } & \multicolumn{2}{|c|}{ Spring } & \multicolumn{2}{|c|}{ Autumn } \\
\hline & Energy $(\mathrm{J})$ & $\operatorname{Pr}$ (fail) & Energy $(\mathrm{J})$ & $\operatorname{Pr}$ (fail) & Energy $(\mathrm{J})$ & $\operatorname{Pr}$ (fail) & Energy $(\mathrm{J})$ & $\operatorname{Pr}$ (fail) \\
\hline p-Sulu & $1.93 \times 10^{4}$ & 0.01 & $3.47 \times 10^{4}$ & 0.00 & $3.37 \times 10^{4}$ & 0.00 & $3.81 \times 10^{4}$ & 0.00 \\
\hline Sulu & $1.65 \times 10^{4}$ & 1.00 & -- & 1.00 & $3.09 \times 10^{4}$ & 1.00 & $3.67 \times 10^{4}$ & 1.00 \\
\hline PID & $3.97 \times 10^{4}$ & 0.00 & $4.17 \times 10^{4}$ & 0.00 & $3.98 \times 10^{4}$ & 0.00 & $3.99 \times 10^{4}$ & 0.00 \\
\hline
\end{tabular}
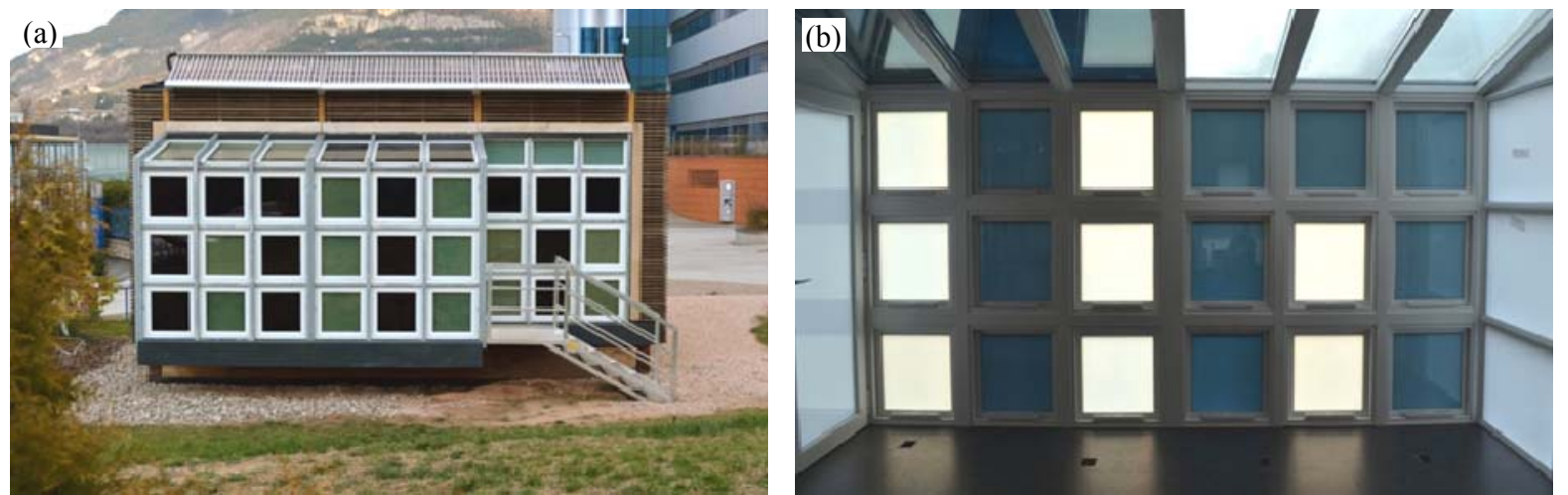

Fig. 12 Visual pattern involving electrochromic and PDLC chromatism variations on the south facade of the prototype, as perceived: (a) from the exterior; (b) from the interior.

The difference in energy consumption of p-Sulu and Sulu is significantly less than that of p-Sulu and the PID controller. But, p-Sulu results in less energy savings compared to Sulu. The cause of this result is that p-Sulu requires operating within safety margins, to satisfy the chance constraints. The extra energy consumption of $p$-Sulu is the price of its robustness. If as a measure of comfort one acknowledges the trials that were completed and considers the fraction of time steps on which a constraint was violated, p-Sulu is the winner. Sulu could not complete any of the summer simulations due to infeasibility of the CCQSP planning problem caused by exogenous disturbances, and therefore the summer energy consumption of Sulu is not included in Table 2. In contrast, p-Sulu is robust to such disturbances because it operates with sufficient safety margins to guarantee that the probability of failure remains always below the given risk bound. The probability of failure of p-Sulu is successfully limited by the given threshold $\Delta$ in all four experiments.

In all, the results of the simulation experiments exhibit that p-Sulu achieves significant energy savings in comparison to a traditional PID controller, while successfully narrowing the probability of failure 
within a given risk bound. Using constraint violations as a measure of success, p-Sulu outperforms the deterministic approach of Sulu. Averaged across all trials, p-Sulu exhibits a difference of $30.88 \%$ in improvement in comfort over Sulu. These results illustrate that risk-sensitive control becomes critical in guaranteeing comfort and encouraging the adoption of the system: A control system that causes uncomfortable conditions $30 \%$ of the time would quickly be abandoned by the users.

\section{Conclusions}

Programmable materials and the application of AI methods to building control can be used to enhance sustainable living and optimize comfort. The features of a programmable facade for a prototype house that was implemented in Trento, Northern Italy were discussed in this paper. These features rest on the capacity to intelligently monitor and modify the state of each individual windowpane of the facade in real time.

Windows are typically operable building components that are essential to the good thermal performance of buildings. The programmable solar facade of the prototype is a matrix of programmable windows. It combines environmentally responsible design with new innovative material and control technologies, and it points to new ways of improving performance and enhancing the quality of everyday life. Each windowpane is an overlay of two electronically switchable, variable transmittance materials. The first electrochromic layer provides the desirable degree of sunlight penetration, securing sunlight and thermal performance. The second PDLC layer provides the desired degree of visibility, securing privacy. The distribution of the active electrochromic windows on the facade may change from one pattern to another while maintaining the same activation ratio, in order to satisfy other user-determined factors, thus animating the way the house is perceived from the public street. Fig. 12 presents an example of a visual pattern involving electrochromic and PDLC chromatism variations on the south facade of the prototype, as perceived: (a) from the exterior; (b) the interior.

At the local level, each windowpane is using low-level intelligence, software and custom electronics that enable the activation of its switchable materials. At the house level, the windowpanes are directed by a central autonomous control system without having interference among them.

The autonomous control system of the prototype aims to optimize the long-term energy performance. It enables the efficient management of the house interior atmosphere through the precise adjustment of the programmable facade. Three words summarize the strengths of the control system, namely comfort, sustainability and convenience: Comfort is achieved by allowing the users to input flexible schedules describing their preferences and activities into the system; sustainability is achieved by minimizing the need for non-renewable energy use; and convenience is ascertained by minimizing the user's effort towards achieving the two previous goals.

A newly developed risk-sensitive plan executive called p-Sulu, capable of optimizing schedule and control sequence with bounded risk of constraint violations is used for the purpose. In addition to providing an optimal plan for the operation of the house, the controller accounts for uncertainty caused by unexpected changes in weather or use-patterns. A probabilistic guarantee that the comfort constraints will not be violated is provided by acknowledging sources of uncertainty and planning accordingly. P-Sulu significantly reduces the energy consumption compared to existing deterministic control methods.

The efficient control of the programmable windows operates in a consistent way to exploit the energy of the sun and the thermal conservation properties of the building envelope. During the hot summer days protecting the interior from direct sun exposure becomes a high priority. Hence, the control sets the programmable windowpanes to their minimum solar transmittance value to effectively block the sun. During the cold winter days exposing the interior to 
the warm winter sun becomes a high priority. Hence, the control sets the windowpanes to their maximum solar transmittance value to permit the storage of heat in the home's passive envelope.

The capacity to reprogram each individual windowpane on the facade has not only functional and performance consequences, but also social and cultural ones. The flexible use modes of the facade range from moderation of view and air, to privacy control and personalized daylight or shade for each window, ultimately promoting personal communication and self-expression. Hence, except from interior climate regulator the facade becomes a responsive mediator between the private and the public domain. It is conceivable that in the future programmable building components, variable transmittance materials and AI methods to building control will attain a higher degree of influence in architecture, giving rise to alternative conceptions of space and eventually to "new architectures", where people will engage in new ways with their living environments.

\section{Acknowledgments}

This research was conducted within the Green Connected Home Alliance between the Mobile Experience Lab, at the Massachusetts Institute of Technology and the Fondazione Bruno Kessler in Trento, Northern Italy.

\section{References}

[1] E.S. Lee, D.L. Di Bartolomeo, J.H. Klems, M. Yazdanian, S.E. Selkowitz, Monitored energy performance or electrochromic windows for daylighting and visual comfort, in: ASHRAE Summer Meeting, Quebec City, Canada, 2006, pp. 122-146.

[2] T. Hausler, U. Fischer, M. Rottmann, K.H. Heckner, Solar optical properties and daylight potential of electrochromic windows, in: International Lighting and Colour Conference, Capetown, 2003, pp. 102-106.

[3] S. Selkowitz, O. Aschehougd, E.S. Lee, Advanced interactive facades-Critical elements for future buildings?, in: U.S. Green Building Council's GreenBuild International Conference and Expo, USA, 2003, http://www.energy.ca.gov/2006publications/CEC-500-20
06-052/CEC-500-2006-052-AT17.pdf (accessed Jan. 1, 2013).

[4] J. Mardaljevic, L. Heschong, E.S. Lee, Daylight metrics and energy savings, Lighting Research and Technology 41 (3) (2009) 261-283.

[5] A.E.D Mady, G.M. Provan, C. Ryan, K.N. Brown, Stochastic model predictive controller for the integration of building use, in: Proceedings of Twenty-Fifth AAAI Conference on Artificial Intelligence (AAAI-11), Special Track on Computational Sustainability and AI, USA, 2011, pp. 1371-1376.

[6] J.Z. Kolter, J Ferreira, A large-scale study on predicting and contextualizing building energy usage, in: Proceedings of Twenty-Fifth AAAI Conference on Artificial Intelligence (AAAI-12), Special Track on Computational Sustainability and AI, USA, 2011, pp. 1340-1356.

[7] H. Li, B.C. Williams, Hybrid planning with temporally extended goals for sustainable ocean observing, in: Proceedings of Twenty-Fifth AAAI Conference on Artificial Intelligence (AAAI-11), Special Track on Computational Sustainability and AI, USA, 2011, pp. 1365-1370.

[8] M. Ono, B.C. Williams, An efficient motion planning algorithm for stochastic dynamic systems with constraints on probability of failure, in: Proceedings of the Twenty-Third AAAI Conference on Artificial Intelligence (AAAI-08), USA, 2008, pp. 1376-1382.

[9] T. Leaute, B.C. Williams, Coordinating agile systems through the model-based execution of temporal plans, in: Proceedings of the Twentieth National Conference on Artificial Intelligence (AAAI), USA, 2005, pp. 114-120.

[10] S. Kotsopoulos, G. Cara, W. Graybill, F. Casalegno, The Dynamic Facade Pattern Grammar, Environment and Planning B: Planning and Design, USA, 2012. (in print)

[11] Annual Energy Outlook 2010, U.S. Energy Information Administration, 2010.

[12] M. Feldmeier, J.A. Paradiso, Personalized HVAC control system, in: Internet of Things (IOT), Tokyo, 2010, pp. 1-8.

[13] M. Ono, Energy-efficient control of a smart grid with sustainable homes based on distributing risk, Master's Thesis, Massachusetts Institute of Technology, 2012.

[14] W. Graybill, Robust, goal-directed planning and plan recognition for the sustainable control of homes, Master's Thesis, Massachusetts Institute of Technology, 2012.

[15] M. Ono, Robust, Goal-directed plan execution with bounded risk, Ph.D. Dissertation, Massachusetts Institute of Technology, 2012.

[16] M. Ono, W. Graybill, B.C. Williams, Risk-sensitive plan execution for sustainable connected home, in: BuildSys'12 Proceedings of the Fourth ACM Workshop on Embedded Sensing Systems for Energy-Efficiency in Buildings, New York, USA, 2012, pp. 45-52. 